\title{
A MONOLITHIC MICRO DEVICE FOR GLUCOSE-RESPONSIVE INSULIN DELIVERY
}

\author{
Jian Chen ${ }^{1}$, Claudia R. Gordijo ${ }^{2} *$, Michael Chu ${ }^{2}$, Xiao $\mathrm{Yu} \mathrm{Wu}^{2} * *$ and $\mathrm{Yu} \mathrm{Sun}^{1} * *$ \\ ${ }^{1}$ Institute of Biomaterials and Biomedical Engineering, University of Toronto, Toronto, Canada \\ ${ }^{2}$ Department of Pharmaceutical Sciences, University of Toronto, Toronto, Canada
}

\begin{abstract}
This paper presents a glucose-responsive device capable of modulating insulin diffusion rates according to changes in environmental glucose levels. A new glucose-responsive composite membrane was developed by crosslinking an albumin-based membrane with a micropatterned PDMS backbone. The membrane was made glucose responsive by the chemical immobilization of glucose oxidase (GOx) and catalase (CAT) along with embedded $\mathrm{pH}$-responsive hydrogel nanoparticles. The nanoparticles act as intelligent 'nano-valves' in response to the enzymatic oxidation of glucose. The membrane responsiveness to glucose concentration was determined by testing the diffusion rates of bovine insulin at alternating glucose levels relevant to diabetic patients. Insulin diffusion across the membrane increases with increasing glucose concentration in the medium, and decreases when glucose is returned to lower levels. The glucose regulated profile of insulin
\end{abstract}

release was also measured on proof-of-concept devices made with PDMS drug reservoirs and integrated glucose-responsive membrane.

\section{INTRODUCTION}

Diabetes is a chronic disease that occurs either when the pancreas does not produce enough insulin or when the body cannot effectively use the insulin it produces. The most effective approach for diabetes management would be a closed-loop insulin delivery system [1]. In this system, a sensing component detects changes of glucose level and produces feedback signals for a releasing component. One limiting factor such a delivery system is the unavailability of a continuous glucose sensor capable of performing fast, accurate measurements with relatively long-term stability for in vivo conditions [2], despite the tremendous efforts in glucose
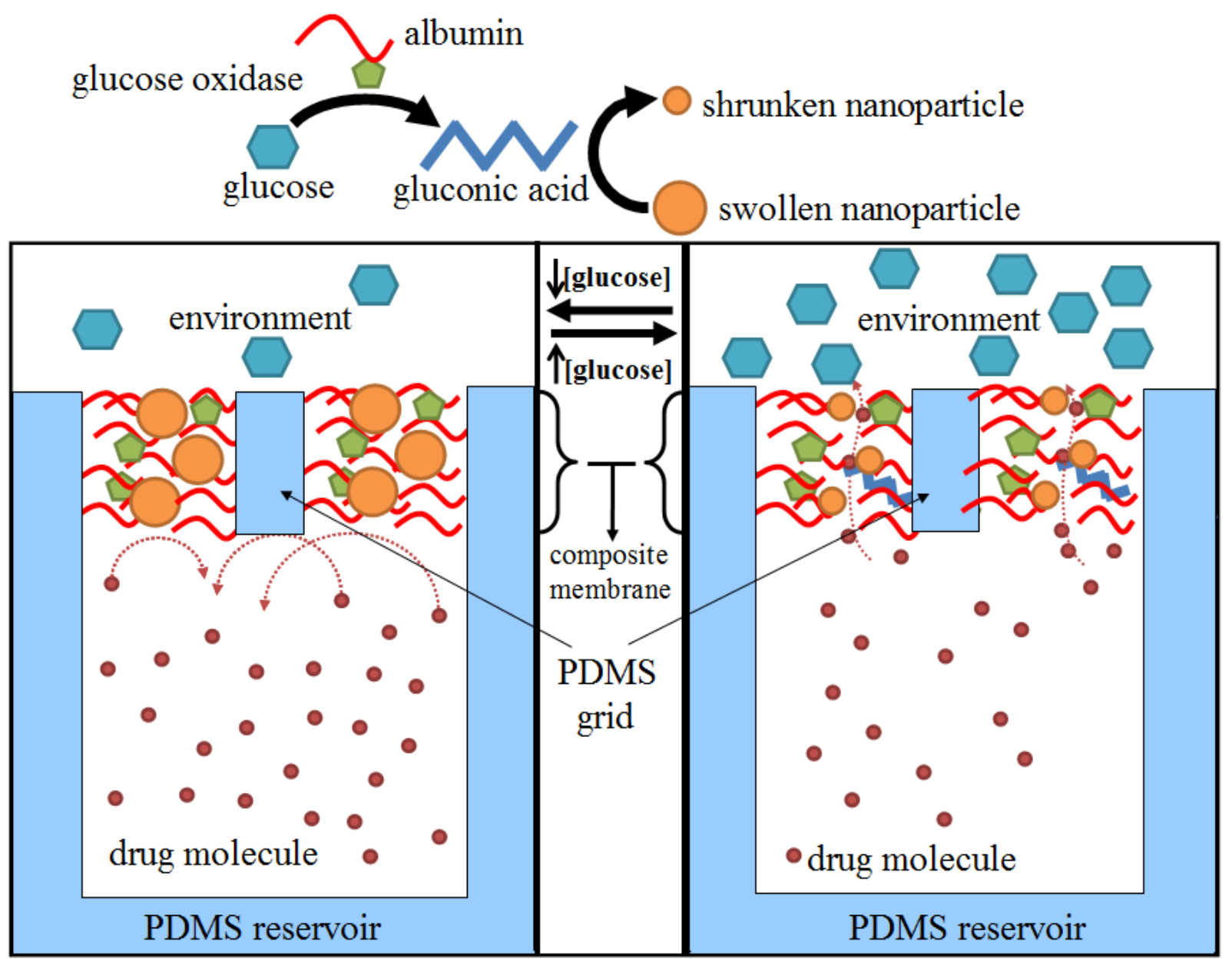

Figure 1: A PDMS drug reservoir holds drug molecules. A layer of PDMS grids on device top provides physical support for the albumin-based composite membrane. Within membrane, enzymes (e.g., glucose oxidase) are chemically crosslinked with the base membrane composed of albumin macromolecules. The embedded hydrogel nanoparticles detect local pH changes caused by gluconic acid resulting from the oxidation of glucose, acting as intelligent nanovalves. Corresponding volumetric swelling and shrinking of the hydrogel nanoparticles in response to environmental glucose values control drug-release rates. 
(a)

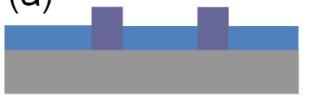

(c)

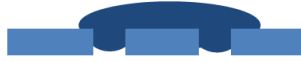

(b)

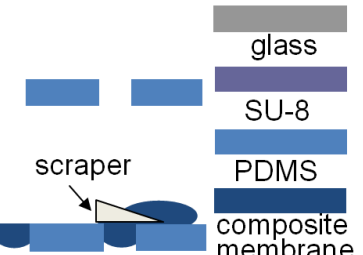

Figure 2: (a) and (b) Standard soft lithography to form PDMS grids. (c) Membrane solution is poured onto the PDMS grids. (d) After removing excess membrane solution using a scraper, the glucose-responsive membrane with PDMS grids as backbone is formed.

sensor development from industrial and academic communities.

Glucose-responsive hydrogels (e.g., combination of enzyme GOx with $\mathrm{pH}$-responsive hydrogels) have been widely applied in the development of self-regulated closed-loop insulin delivery systems [3]. Since transient behavior of the hydrogel is limited by the diffusion of glucose into the hydrogel matrix and by the absorption and expulsion of solvent, glucose-responsive hydrogels at smaller scales have been developed for controlled drug delivery with shorter response times [4,5].

Previously, controlled release of drugs was achieved by microfabricated devices via electrochemical dissolution, electrothermal activation or chemical degradation approaches [6-8]. Despite the on-demand drug release capability, these implantable drug-delivery micro devices are not able to self-regulate the drug delivery rates in response to local micro-environmental signals, such as $\mathrm{pH}$ and glucose changes.

We previously demonstrated proof-of-principle glucose-responsive insulin release through a composite membrane consisting of a hydrophobic base membrane containing embedded pH-responsive hydrogel nanoparticles, GOx, and CAT [5]. We recently developed a new glucose-responsive membrane by introducing bio-inorganic nanohybrid composite and chemical crosslinking of albumin with the enzymes [9]. This new nanohybrid material has been successfully applied as a glucose-responsive plug in a small prototype implantable device for a controlled insulin delivery system [10]. However, owing to the soft nature of this membrane, the material would not be suitable for use as a free membrane in relatively large areas.

In the present work, we propose (1) the application of micro patterned PDMS grids to support the albumin-based composite membrane to enhance its mechanical strength; and (2) the design of a monolithic glucose-responsive drug-delivery micro device by integrating the composite membrane and a micro drug reservoir.

\section{DESIGN AND FABRICATION}

\section{General considerations about the device's mechanism}

(a)
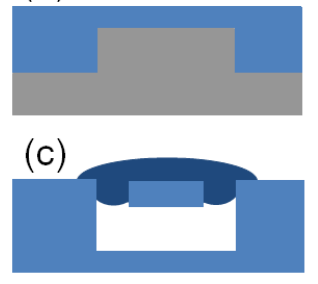

(b)

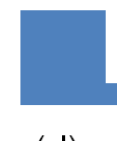

(d)
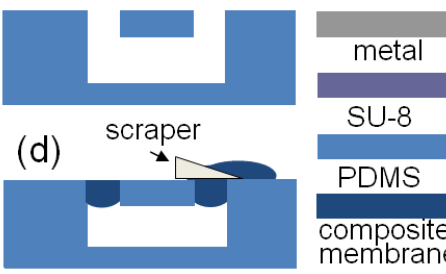

composite membrane

Figure 3: Microfabrication steps for forming glucose-responsive micro devices with enhanced mechanical strength
As shown schematically in Fig. 1, a PDMS drug reservoir and a layer of PDMS grid provided physical support for the composite membrane. In this composite membrane, the enzymes GOx and CAT are directly crosslinked with the albumin macromolecules, forming the base membrane. The embedded hydrogel nanoparticles detect and respond to local $\mathrm{pH}$ changes caused by gluconic acid resulted from the oxidation of glucose by GOx, acting as intelligent nanovalves. Corresponding volumetric swelling and shrinking behaviors of the hydrogel nanoparticles controls the porosity of the membrane, leading to lower or higher drug release rates according to the glucose concentration in the medium.

\section{Preparation of glucose-responsive composite membranes with PDMS grid backbone}

To fabricate glucose-responsive membranes with a PDMS backbone, SU-8 pillars of $500 \times 500 \times 250 \mu \mathrm{m}^{3}$ were formed via photolithography (Fig. 2(a)), as a mold master by spinning SU-8 2100 at 1000 RPM. PDMS was spin coated on the SU-8 mold master at $1000 \mathrm{RPM}$, and fully cured PDMS membrane with a thickness of $150 \mu \mathrm{m}$ was peeled off from the substrate, producing a PDMS grid (Fig. 2(b)).

The surface of the PDMS grid was activated with oxygen plasma, prior to the application of the glucose-responsive membrane solution on grids' surface. The glucose-responsive albumin-based membrane was prepared as previously described [10] by the crosslink of BSA and $\mathrm{MnO}_{2}$ nanoparticles (10 wt \%) with glutaraldehyde in the presence of the $\mathrm{pH}$-responsive hydrogel nanoparticles $(30 \mathrm{wt} \%)$ and the enzymes GOx (5 wt \%) and CAT (1.6 wt \%).

In contrast to our previous work where the membrane was allowed to crosslink on a glass surface, we apply a homogenous mixture of nanoparticles, enzymes, crosslinker and albumin in aqueous solution onto the PDMS grid after surface activation (Fig. 2 (c)). After removing the excess of membrane solution from the top of the grids, the remaining solution between the grids continues to crosslink, forming a glucose-responsive membrane strengthened by the PDMS backbone (Fig. 2(d)).

\section{Fabrication of glucose-responsive micro devices}

To fabricate glucose-responsive micro devices, firstly a PDMS reservoir was made via standard soft lithography using a metal mold master with a thickness of $2 \mathrm{~mm}$ (Fig. 3(a)). After the PDMS drug reservoir was peeled off from the mold master, the PDMS grid described earlier was bonded with the drug reservoir via a thin layer of pre-cured PDMS as an adhesive layer (Fig. 3 (b)). The mixture of the composite membrane solution was then cast on the PDMS grid as described in the previous section (Fig. 3 (c) and (d)).

Fig. 4(a) shows a 3-D model of the device. Pictures of the prototype devices are shown in Fig.4 (b). The drug reservoirs were constructed with PDMS due to its mechanical stability, the feasibility of precise patterning using microfabrication, and its (a)

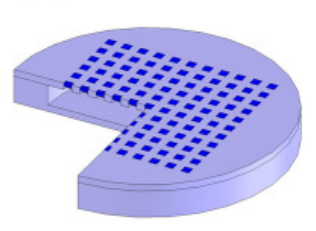

(b)

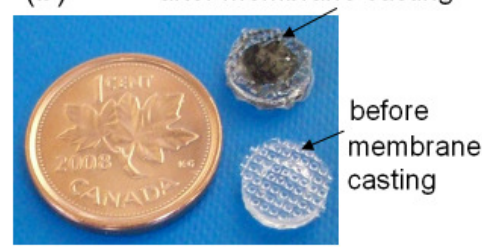

Figure 4: (a) 3D model of the device. (b) Prototypes of fabricated glucose-responsive micro devices. 
(a)

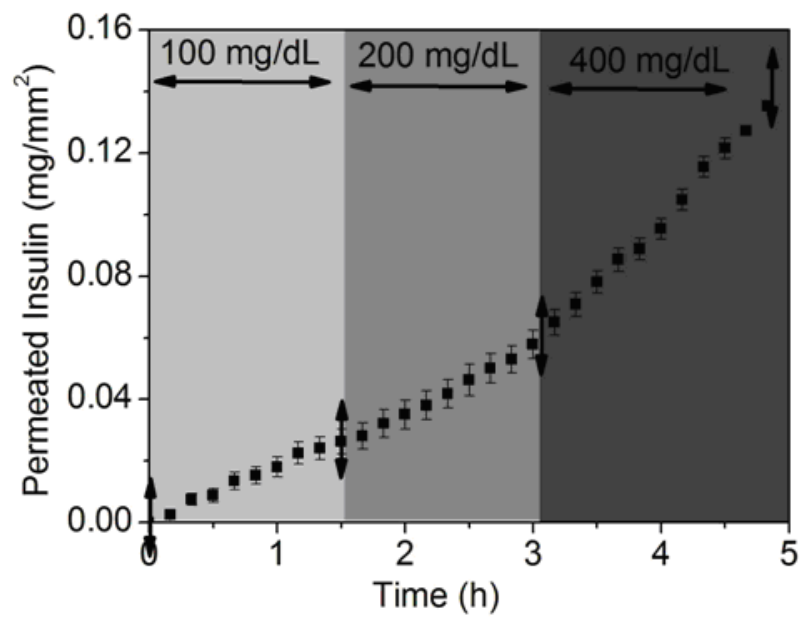

(b)

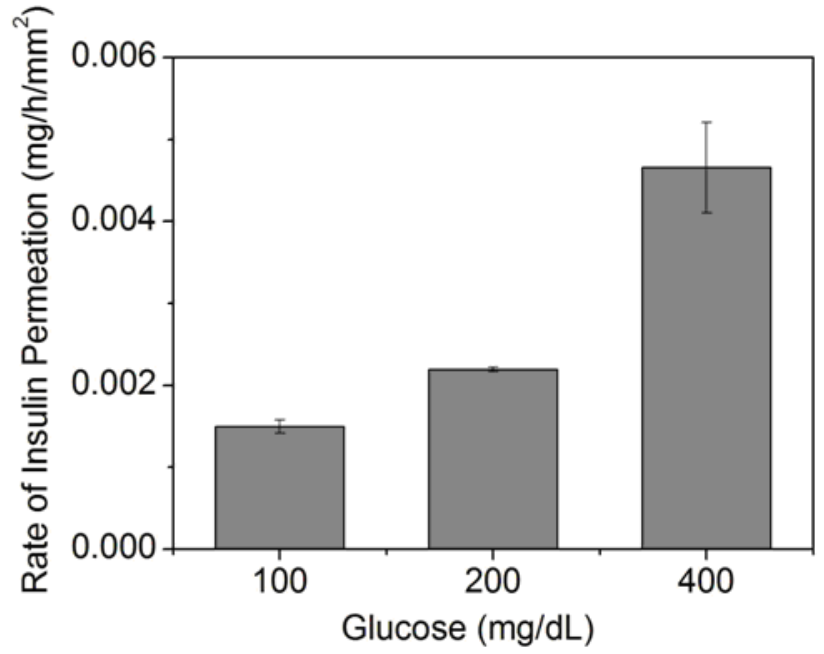

Figure 5: Glucose-responsive permeation of insulin through microstructured membranes $(n=3)$ with integrated PDMS backbone. (a) Profile of insulin permeated across the membrane in response to changes of step-wise glucose concentration (100, 200, and 400 mg/dL, respectively). (b) Permeability of insulin as a function of glucose concentration calculated from the slopes of the curves. Errors bars represent standard deviation.

short-term biocompatibility. Other biocompatible materials such as PEVA, poly(ethylene-co-vinyl acetate) are also viable options. Bovine insulin solution ( $10 \mathrm{mg} / \mathrm{mL}$ in HEPES buffer, $\mathbf{M}_{\mathrm{w}}$ 5808) was used to fill the devices. Insulin filling and refilling was conducted through the backside of the PDMS reservoir with a small-gauge syringe needle, utilizing the self-sealing property of PDMS.

\section{EXPERIMENTAL RESULTS}

\section{Insulin permeation testing of glucose-responsive membranes}

The permeation of insulin $\left(P_{I}\right)$ through the membrane was determined as a function of time and glucose concentration using a horizontal side-by-side diffusion cell system at controlled temperature $\left(37^{\circ} \mathrm{C}\right)$ as we previously described [10]. Briefly, the membrane was placed between two cells: a donor cell containing insulin in pH 7.4 PBS buffer (insulin $2 \mathrm{mg} / \mathrm{mL}$, phosphate $10 \mathrm{mM}$, $\mathrm{NaCl}$ 0.15M, Pluronic F-68 $0.02 \mathrm{mM}$ and glucose $100 \mathrm{mg} / \mathrm{dL}$ ) and a receptor cell filled with the same solution but without insulin (release medium). After $1.5 \mathrm{~h}$ and $3 \mathrm{~h}$ the glucose concentration in the cells was increased to $200 \mathrm{mg} / \mathrm{dL}$ and $400 \mathrm{mg} / \mathrm{dL}$, respectively, by adding aliquots of highly concentrated glucose solution (20 $\mathrm{g} / \mathrm{dL}$ ). During the course of the experiment the release medium was continually pumped through a UV-flow cell, and the insulin permeation was determined by measuring insulin absorbance at 10 min intervals at $\lambda=276 \mathrm{~nm}$ using an UV spectrophotometer. The rate of insulin permeation was determined by calculating the slope of the curves. Fig. 5 shows a representative set of permeability testing data, which demonstrates the glucose responsiveness of the composite membrane. As shown in the figure, when the glucose concentration in the medium was increased from normal (100 $\mathrm{mg} / \mathrm{dL})$ to hyperglycemic levels $(200-400 \mathrm{mg} / \mathrm{dL})$ the insulin permeation rate increased from $0.0015 \mathrm{mg} / \mathrm{h} / \mathrm{mm}^{2}$ to 0.0022 $\mathrm{mg} / \mathrm{h} / \mathrm{mm}^{2}$ and $0.0046 \mathrm{mg} / \mathrm{h} / \mathrm{mm}^{2}$, respectively (1.5-fold and 3.1-fold increase in insulin permeation, respectively, as determined by the relative insulin permeation rates $P_{I-200} / P_{I-100}$ and $\left.P_{I-400} / P_{I-100}\right)$.

\section{Insulin release testing of glucose-responsive devices}

Insulin release from the microdevices was measured as a function of time and glucose concentration in $\mathrm{pH}$ 7.4 PBS buffer containing Pluronic F-68 $(0.02 \mathrm{mM})$, at $37^{\circ} \mathrm{C}$. Microdevices filled with bovine insulin (solution $10 \mathrm{mg} / \mathrm{mL}$ ) were individually placed in vials containing $5 \mathrm{~mL}$ of $100 \mathrm{mg} / \mathrm{dL}$ glucose in the PBS medium. Vials were sealed and kept under constant mixing in a mini blot mixer. No leakage of insulin via the interface between composite membranes and PDMS drug reservoirs was observed in the in vitro insulin release tests of the devices. After two hours, glucose concentration was increased from $100 \mathrm{mg} / \mathrm{dL}$ (normal glucose level) to $300 \mathrm{mg} / \mathrm{dL}$ (hyperglycemic glucose level). The same procedure was repeated for testing each device. The released insulin was determined by measuring insulin absorbance manually every $30 \mathrm{~min}$ at $\lambda=276 \mathrm{~nm}$ using what using the UV spectrophotometer. The rate of insulin release was determined by calculating the slope of the curves.

Fig. 6 shows insulin release profiles of two-cycle tests for four microdevices. A 2.4-fold increase in the insulin release rate was observed when the glucose concentration was increased to hyperglycemic levels (from $100 \mathrm{mg} / \mathrm{dL}$ to $300 \mathrm{mg} / \mathrm{dL}$ ). Additionally, the insulin release rate decreased to the initial rate $\left(\sim 0.0030 \mathrm{mg} / \mathrm{h} / \mathrm{mm}^{2}\right)$ when the glucose concentration was decreased to the normal level $100 \mathrm{mg} / \mathrm{dL}$ in the second cycle. This behavior demonstrated the device's capability for regulated release.

\section{CONCLUSION}

This paper demonstrated a new type of monolithic glucose-responsive drug-delivery device. The microdevices integrated glucose-responsive composite membranes with PDMS backbones in which $\mathrm{pH}$-responsive nanoparticles were embedded and glucose oxidase was chemically crosslinked. Diffusion testing demonstrated the capability of these devices for glucose-responsive insulin release, with an increase in release rates in response to increasing glucose levels. This device concept may serve as a platform technology for intelligent drug delivery in response to environmental glucose variations. Further work will continue to optimize and fine tune the composite membrane parameters as well as the quantification of insulin stability and delivery kinetics in vitro and in vivo. 
(a)

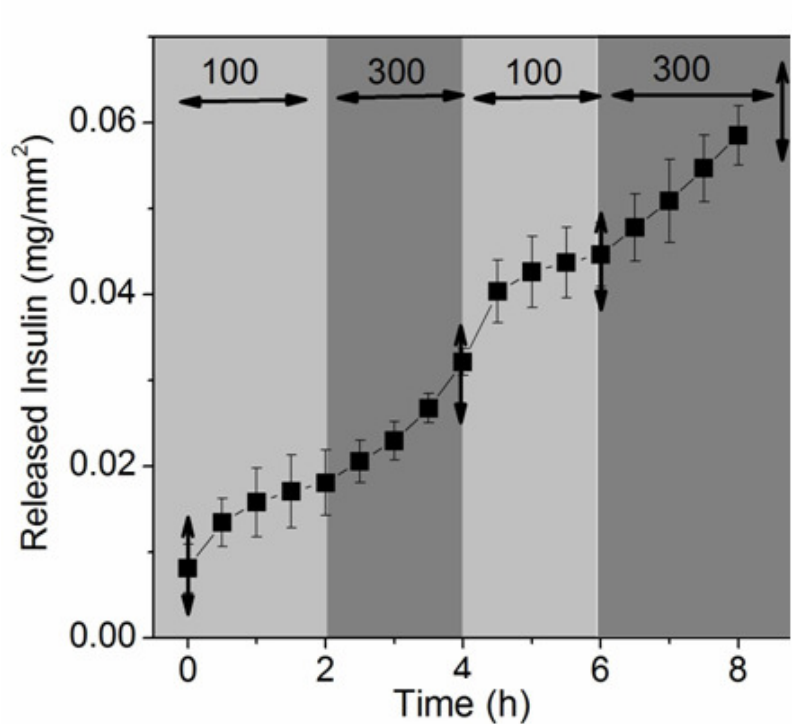

(b)

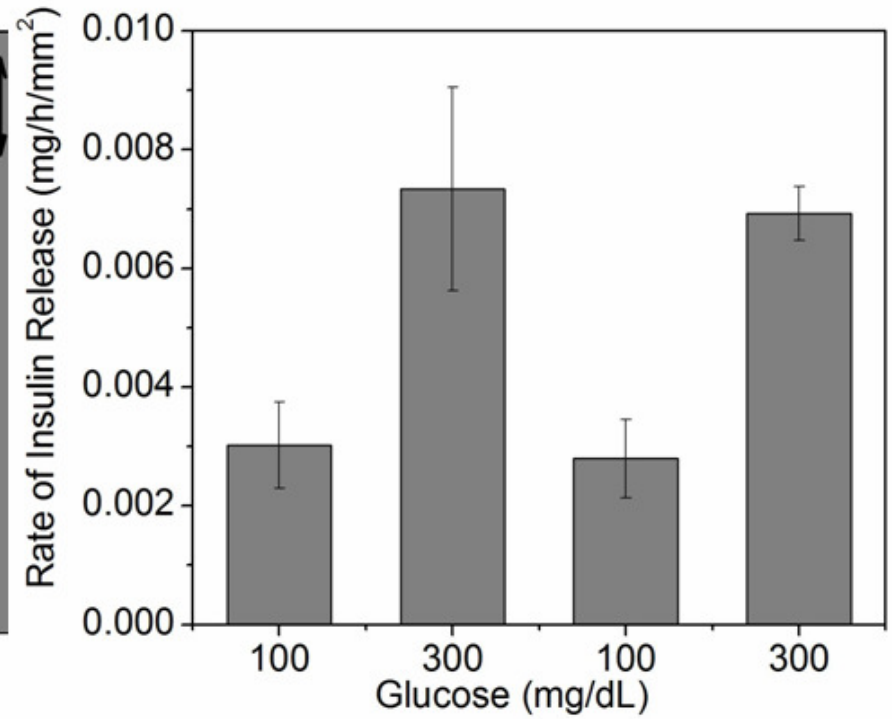

Figure 6: (a) Profile of insulin released from micro devices in response to changes in glucose concentration in two alternated cycles $(n=4)$. Glucose concentration was alternated between $100 \mathrm{mg} / \mathrm{dL}$ and $300 \mathrm{mg} / \mathrm{dL}$ to simulate changes in blood glucose levels of diabetic patients (normal and hyperglycemic levels, respectively). (b) Insulin release rates of the micro devices as a function of glucose concentration. Error bars represent standard deviation.

\section{REFERENCES}

[1] M.Shichiri et al., "Enhanced Simplied Glucose Sensors Long-term Clinical Application of Wearable Artifical Endocrine Pancreas", Artifial Organs, 22, 32 (1998).

[2] R. Hovorka, "Continuous Glucose Monitoring and Closed-loop Systems", Diabetic Medicine, 23, 1 (2006).

[3] E.Renard et al., "Closed Loop Insulin Delivery Using Implanted Insulin Pumps and Sensors in Type 1 Diabetic Patients", Diabetes Research and Clinical Practice, 74, 173 (2006).

[4] R. Siegel, B. Ziaie et al., "A Hydrogel-Actuated Environmentally Sensitive Microvalve for Active Flow Control", J. Microelectromechanical System, 12, 613 (2003).

[5] K. Zhang, X.Y.Wu, "Modulated Insulin Permeation Across a Glucose-sensitive Polymeric Composite Membrane", J. Control Release, 80, 169 (2002).

[6] R. Langer et al., Nature, "A Controlled-release Microchip", Nature, 397, 335 (1999).

[7] J. Prescott et al., "Chronic, Programmed Polypeptide Delivery from an Implanted, Multi-reservoir Microchip Device", Nature Biotechnology, 24, 437 (2006).

[8] R. Langer et al., "Multi-pulse Drug Delivery from a Resorbable Polymeric Microchip Device", Nature Material, 2, 767 (2003).

[9] C.R. Gordijo, A.J. Shuhendler, X.Y. Wu, "Glucose-responsive Bio-inorganic Nanohybrid Membrane for Self-regulated Insulin Release", Advanced Functional Materials, In press (2010).

[10] C.R.Gordijo, K.Koulaijan, A.Giacca, X. Y. Wu, "Closed-Loop Insulin Delivery Device: Fabrication, In Vitro and In Vivo Evaluation of Self-Regulated Insulin Release", Diabetes Technology Meeting, San Francisco, USA (2009), pp A188.

\section{ACKNOWLEDGEMENTS}

This work was supported by a NSERC-CIHR Collaborative Health Research Grant (CHRP\#482996).

\section{* THOSE AUTHORS CONTRIBUTED EQUALLY **CO-CORRESPONDING AUTHORS:}

Xiao $\mathrm{Yu} \mathrm{Wu}$, Leslie Dan Faculty of Pharmacy, University of Toronto, 144 College Street, Toronto, Canada M5S 3M2. Tel: 1-416-978-5272; Email:sxy.wu@utoronto.ca

Yu Sun, Institute of Biomaterials and Biomedical Engineering, University of Toronto, 5 King's College Road, Toronto, Canada M5S 3G8. Tel: 1-416-946-0549; E-mail: sun@mie.utoronto.ca 